\title{
Inestabilidad de Beta de Sectores Económicos en la Bolsa de Comercio de Buenos Aires (1994-2007)
}

\author{
Mauro Ferraro* \\ Tesis de Maestría en Economía \\ Universidad Nacional de La Plata
}

19 de septiembre de 2008

\begin{abstract}
Resumen
Si bien el CAPM no requiere que beta sea estable en el tiempo, al trabajar con series de datos y estimar su valor en el contexto del Modelo de Índice Simple, la estabilidad del coeficiente se torna en una condición crucial para su adecuada utilización. Una práctica ampliamente difundida consiste en obtener los valores a través de MCO, asumiendo la estabilidad de dicho coeficiente. El presente trabajo estima los coeficientes beta de portafolios de sectores económicos con oferta pública de acciones en la Bolsa de Comercio de Buenos Aires en el período 1994-2007, introduciendo una metodología de estimación no paramétrica denominada Varying Coefficient Model. El ejercicio muestra la importante volatilidad de los betas, siendo que es por ello altamente recomendable tomar con especial cuidado las estimaciones de betas basadas en datos históricos al querer extrapolarlas en el tiempo. La utilización en esta dirección, puede modificar drásticamente las conclusiones en la práctica de la administracción de portafolios de inversión y en la valuación de empresas. Dos ejemplos de estas aplicaciones son mostradas en el anexo.
\end{abstract}

Palabras Clave: Beta, CAPM, Estabilidad, Retorno, Econometría no Paramétrica, Sectores Económicos.

Códigos JEL: G11, G12, G14.

*Agradezco los comentarios de Ricardo Bebczuk (UNLP, UDESA y BCRA), José Dapena (UCEMA), Alejandro Bour (UADE y ROFEX), Damián Sainz de Aja (Nación AFJP), Ricardo Pasquini (UTDT y CEF) y María Laura Oliveri (UNLP). Así también la colaboración de Maik Eisenbeiss (Universität Münster). 


\section{Introducción}

Sin dudas, una de las contribuciones académicas más importantes en el campo de las finanzas, fue derivar un modelo de equilibrio general que determine la medida relevante de riesgo de cualquier activo y la relación entre retorno esperado y riesgo cuando los mercados están en equilibrio. Esto es el resultado del Capital Asset Princing Model (CAPM), derivado independientemente por Sharpe (1964), Lintner (1965) y Mossin (1966), y contruído a partir de Markowitz (1952).

Dada una tasa libre de riesgo ${ }^{1}$, cada agente averso al riesgo, tal como advierte el Principo de Separación (Tobin 1958), arma posiciones en base a sus preferencias por el riesgo. Asumiendo que todos los inversores conocen como se distribuyen los retornos entre $t-1$ y $t^{2}$, enfrentan el mismo conjunto de oportunidades y combinan el mismo portafolio de activos riesgosos, denominado portafolio de mercado $^{3}$, prestando o tomando prestado a la tasa de interés libre de riesgo.

Dentro del portafolio de mercado, la medida de riesgo de un activo individual no es la varianza de sus retornos, sino la manera en que contribuye al riesgo del portafolio de mercado. De esta manera, el riesgo de un activo individual depende de su exposición a lo que ocurre en el mercado. Así, beta puede interpretarse como la sensibilidad del activo individual al riesgo de mercado ${ }^{4}$, y representa una medida estandadizada en 1 , de la covarianza entre los retornos del activo individual y del mercado.

El modelo representa la relación entre el retorno esperado y beta:

$$
R_{j=} R_{F}+\beta\left(R_{M}-R_{F}\right)+\varepsilon_{j}
$$

Donde $R_{j}$ es el retorno esperado del activo individual, $R_{M}$ el retorno esperado del mercado, $R_{F}$ es el retorno libre de riesgo y $\varepsilon_{j}$ refleja las variaciones en el retorno debido a cambios que son idiosincrásicos. Este componente debe entenderse como la parte del retorno del activo individual que no está correlacionado con el retorno del mercado. Debido a que esta fracción de riesgo puede ser eliminada por un inversor que invierte óptimamente en el portafolio de mercado, no

\footnotetext{
${ }^{1}$ Esta tasa es simplemente la de equilibrio entre ofertantes y demandantes de fondos.

${ }^{2}$ La función de distribución es asumida normal, o bien cualquier otra siempre que la función de utilidad, que están maximizando los inversores, sea cuadrática.

${ }^{3}$ Es aquel que contiene todos los activos riesgosos, de manera que cada uno de ellos es incluido de acuerdo a la proporción de valor de mercado que representa en el valor de mercado de la totalidad de los activos riesgosos.

${ }^{4}$ Técnicamente, el beta de un activo es el cociente entre la covarianza del retorno del activo y del mercado, y la varianza del mercado: $\beta_{j}=\frac{\operatorname{Cov}\left(R_{j} R_{M}\right)}{\operatorname{Var}\left(R_{M}\right)}$. En otros términos, es la covarianza del activo individual en el mercado, relativo a la covarianza promedio de los activos, la cual es la varianza del retorno del mercado. Mientras que el riesgo del portafolio de mercado (su varianza) es el promedio ponderado de los pares de covarianzas de los activos dentro de él.

Por definición, el mercado tiene beta igual a uno, dado que el beta promedio ponderado de todos los activos, ponderando por la porción del valor de cada activo en el valor del mercado, es $1: \sum_{i} x_{i} \beta_{i}=1$.
} 
tiene premio. De hecho, el modelo especifica que solamente la porción del riesgo que no es diversificable, es recompensada.

El segundo término de la derecha representa el premio por riesgo del activo $i$ : el premio por unidad de su beta. Como apuntan Brealey, Myers y Marcus (2001) la idea central del CAPM es que los inversores esperan una recompensa por esperar (primer término de la derecha de 1) y por estar preocupado (segundo término).

Fama y MacBeth (1973) testearon tres implicancias del modelo CAPM: $i$ ) la linealidad implícita en el concepto de arbitraje y eficiencia de mercado ${ }^{5}$; los inversores no van a dejar sistemáticamente activos sub o sobre valuados ${ }^{6}, i i$ ) que las diferencias entre los retornos de los activos y el mercado es enteramente explicado por las diferencias en beta, siendo que ninguna otra variable adiciona valor explicativo, y iii) que en un mercado con inversores aversos al riesgo, mayor riesgo debe ser asociado con mayor retorno esperado. Los autores concluyen, utilizando datos de la Bolsa de Nueva York, que la relación entre el riesgo de un portafolio de activos y su retorno es lineal, que no es necesaria ninguna medida adicional de riesgo para explicar el retorno del activo individual más allá del retorno del mercado, y que, en promedio, existe un trade-off positivo entre riesgo y retorno. En este punto, Bandi y Perron (2008) también encuentran este tradeoff pero con rezagos. Activos con varianza grande en el pasado están altamente correlacionados con mayor retorno en el futuro, más aún a largo plazo ${ }^{7}$.

No obstante, una serie de trabajos posteriores demuestran que cocientes que involucran precios de los activos, como price-earning, capitalización bursátil, apalancamiento y book-to-market ratio, guardan información acerca del retorno esperado, omitido por beta (Basu 1977, Banz 1981, Bhandari 1988, Fama y French 1992, 1996a).

Campbell, Lo y MacKinlay (1997) señalan que si bien las anomalías encontradas por la literatura aportan importantes desvíos del CAPM, hay poca motivación teórica para ello. Además, las anomalías descubiertas pueden ser exageradas debido a que pueden tener su origen en problemas en la recolección y procesamiento de los datos.

Otra serie de trabajos apuntan a los supuestos del modelo en cuanto a que el riesgo es medido por la varianza en vez de la semivarianza (Hogan y Warren 1974, Bawa 1975, Bawa y Linderberg 1977, Price, Price y Nantell 1982, Pedersen y Hwang 2007) ${ }^{8}$.

\footnotetext{
${ }^{5}$ La hipótesis de eficiencia del mercado establece que el precio de los activos refleja toda la información disponible.

${ }^{6}$ Cualquier activo con retorno esperado por sobre o por debajo de la Línea del Mercado de Capitales, es corregido por arbitraje vendiendo o comprando short o long.

${ }^{7}$ En el largo plazo, por ejemplo unos 10 años, la varianza pasada es un importante predictor del exceso de retorno en el contexto del CAPM.

${ }^{8}$ El modelo de media-semivarianza, considera solamente la dispersión en el lado de las pérdidas tomando como referencia un cierto nivel de retorno. Bawa (1975) sienta las bases teóricas y propuso cambiar la medida de beta por una de downside beta, en base al concepto de dominancia estocástica. Bawa y Linderberg (1977) desarrollaron una medida de riesgo sistemático que depende de las variaciones del retorno del activo solamente debajo de la tasa libre de riesgo. Así, derivan un modelo alternativo de valuación al CAPM, al igual que Hogan
} 
Por décadas, el CAPM ha sido ampliamente utilizado en aplicaciones financieras como estimar el costo de capital de firmas, valuación de empresas, evaluación de proyectos y administración de portafolios. Sin embargo, el modelo cuenta con problemas empíricos. Justamente, si bien el modelo no requiere que el beta del activo individual sea estable, presume que los agentes siempre conocen su valor. El uso del modelo es el que requiere que los coeficientes sean estables (Coutts, Roberts y Mills 1997). De hecho, la interpetación de beta como medida de riesgo descansa sobre la validez empírica del modelo.

Una práctica usual consiste en estimar dicho coeficiente corriendo una regresión del retorno realizado por el activo contra el retorno realizado por el mercado. Alternativas de estimación como Mínimos Cuadrados Ordinarios (MCO) asumen que beta es estacionaria. En este caso, la validez de usar beta como medida de riesgo sistemático depende del supuesto de que el mismo es estacionario en el tiempo (Chen 1981).

El objetivo de este trabajo es estudiar la estabilidad de los beta de portafolios de sectores que tienen sus respectivas empresas con oferta pública en la Bolsa de Comercio de Buenos Aires (BCBA) durante los años 1994 y 2007, similarmente a lo hecho por Eisenbeiss, Kauermann y Semmler (2007) para la Bolsa Alemana para el período 1991-2003. La idea central constituye en relajar la estructura de relación, modelando a beta como un proceso más que como un coeficiente fijo. En especial, el aporte a la literatura consiste en introducir un término de interacción entre el retorno de mercado y el tiempo. Mediante una técnica de estimación no paramétrica, conocida como el Varying Coefficient Model, es posible poner en juicio la estabilidad de beta en el tiempo, aplicado al mercado local. Además de la contribución a la academia, tanto la metodología aplicada como las conclusiones del trabajo esperan servir como una importante guía a administradores de portafolios, analistas de valuaciones de activos financieros, evaluadores de proyectos e inversores en general.

El trabajo se organiza como sigue: la sección siguiente resume los principales trabajos que se han encargado de estimar betas y su estabilidad. La sección tercera se encarga de introducir los conceptos del herramental econométrico utilizado. La sección cuarta está dedicada a los datos utilizados, la construcción de los portafolios, comentarios adicionales sobre los datos y la presentación de estadísticas descriptivas. La sección quinta estudia la estabilidad de los coeficientes mediante la especificación del modelo descripto. La sección sexta presenta las conclusiones generales. Por último, en el anexo se exponen posibles aplicaciones en la administración de portafolios de inversión y en las valuaciones de empresas.

y Warren (1974). Price, Price y Nantell (1982) presentan un teorema que concluye que las dos medidas de riesgo difieren cuando se asume una distribución bivariada lognormal entre los retornos del activo individual y el mercado, no así cuando las distribuciones son normales. Pedersen y Hwang (2007) trabajaron con datos de la bolsa londinense y concluyeron que el modelo basado en el concepto de semivarianza explica un poco mejor los retornos de las acciones. 


\section{Revisión de Literatura}

Llevar a cabo una confección eficiente de un portafolio de activos, requiere contar con el retorno de cada activo y la matriz de varianzas y covarianzas, es decir la varianza de cada activo y la covarianza entre todos los pares de activos. Como todos los activos que cotizan en el mercado son candidatos de ser incluidos en el portafolio, el problema se torna complejo ya que requiere la estimacion de: $n+\frac{n(n-1)}{2}$ coeficientes $^{9}$. Una interesante alternativa la constituye el Modelo de Índice Simple (MIS), partiendo de la premisa que no hay efectos más allá del mercado, medido por beta, que afecten significativamente la dinámica del precio, y así del retorno del activo en cuestión ${ }^{10}$.

No solamente para las decisiones de portafolio es necesario contar con los betas. En las evaluaciones de proyectos se requiere de una adecuada tasa de descuento. En la estimación del costo de financiamiento también el hecho crucial pasa por estimar los betas. Por ejemplo, para hallar el rendimiento exigido por los inversores de un activo, basta con conocer su beta, dado que tanto la tasa libre de riesgo como el premio por riesgo pueden obtenerse, como aproximación, a partir de datos históricos.

En la práctica, los individuos se ven obligados a usar datos históricos para estimar el riesgo de los activos. Este hecho torna crucial determinar si los betas pueder ser intertemporalmente estacionarios (Kryzanowski y To 1984). Elton y Gruber (1995) señalan que hay dos razones para estimar betas: directamente para predecir el beta futuro y, por otro lado, para generar los coeficientes de correlación a ser usados en los problemas de elección de portafolio.

Levy (1971) fue unos de los primeros en advertir sobre la no estacionariedad de beta, señalando que el beta de un sólo activo financiero en un período, no es un buen predictor del correspondiente beta, para el mismo activo, en el período siguiente. Blume (1971) encontró que los betas tendían a regresar a la media en el tiempo, sobretodo en portafolios conservadores. Expuso que, como ninguna variable económica es constante en el tiempo, tampoco beta lo es, aunque no hay ningún impedimento para que se la utilice creyendo que es estacionaria. Baesel (1974) concluyó que la estabilidad de beta depende del intervalo de tiempo usado en la estimación.

Blume (1975) investigó cuán bien los betas estimados en un período predecían los comovimientos futuros de ciertos activos con el mercado. Básicamente, comparó betas estimados en diferentes períodos, no superpuestos, evidenciando que los betas tienden a converger hacia la media del de mercado. Desde que beta del portafolio de mercado es el promedio ponderado de todos los betas, activos con betas altas o bajas tienden a tener un beta aproximándose a uno en el futuro. Es por ello que aporta una metodología de ajuste en dos etapas que

\footnotetext{
${ }^{9}$ Por ejemplo, a fines de 2007 cotizan 111 acciones en la BCBA. Con lo que los agentes habrían de estimar 6216 coeficientes.

${ }^{10}$ Es decir, el comovimiento con el mercado es el que determina que dos activos se muevan juntos.
} 
cuente por este hecho ${ }^{11}$.

Alexander y Chervany (1980) también encuentran evidencia de que la tendencia de betas de los activos tiende a ser uno. Blume (1975) atribuyó que la no estabilidad reside en que existen factores propios en los proyectos que encara la firma entre un período y otro. Justamente en un trabajo anterior, Beaver, Kettler y Scholes (1970) habían confiado en variables que capturaban atributos de los fundamentales de las firmas para explicar su riesgo. Es decir, como el beta es una medida del riesgo devenida de la relación entre el retono del activo y el del mercado, bien puede suceder que el riesgo de la firma esté determinado tanto por las características del mercado como por sus fundamentales ${ }^{12}$. No obstante, como señalan Elton y Gruber (1995), la desventaja de esta metodología es que refleja cambios en las características de la compañía solamente después de un tiempo, pudiendo subestimar el impacto sobre el beta computado a partir de datos históricos. No obstante, esta línea ha sido seguida por otros autores aunque con resultados contrapuestos (Breen y Lender 1973, Gonedes 1973, Melicher 1974, Rosenberg y McKibben 1973).

Klemkosky y Martin (1975) sugirieron una combinación de predictor bayesiano, al igual que Vasicek (1973), Beaver y Manegold (1975) y Chen (1981), junto a un tamaño grande de portafolio para ganar precisión en la extrapolación a futuro de betas estimados con datos históricos. Sunder (1980) aporta evidencia de la no estacionariedad de beta en períodos largos (1926-1975).

Fabozzi y Francis (1977) estudiaron los coeficientes en contextos de mercados alcistas y bajistas, en consonancia con las premisas de Levy (1974) y Black (1972). Sin embargo, no encuentran una afección significativa entre los coeficientes en uno u otro momento del mercado. No obstante, en Fabozzi y Francis (1978) aportaron evidencia de que muchas betas se mueven aleatoriamente en el tiempo más que permanecer constantes. En este sentido, Bos y Newbold (1984) rechazan, mediante un test de multiplador de Lagrange, la hipótesis de la estabilidad del coeficiente en pos de que el riesgo sistemático es estocástico.

Kalay y Loewenstein (1985) señalaron que el riesgo es mayor en épocas de anuncios de pagos de dividendos. A esto llegaron haciendo uso de tests de diferencia de medias sobre los retornos y del supuesto de eficiencia del mercado ${ }^{13}$. Además, como este riesgo es en parte no diversificable, el beta es mayor durante el período de pago de dividendos.

Conn (1985) estudió el CAPM en contextos de fusiones de firmas, señalando la inestabilidad de beta debida a cambios en los riesgos operativos involucrados

\footnotetext{
${ }^{11}$ Calcular los betas para el primer y segundo período por separado, para luego regresar los betas de un período sobre el del otro. Luego utilizar los coeficientes estimados para corregir los betas de un tercer período.

${ }^{12}$ Usaron como regresores los dividendos pagados, el crecimiento de los activos, el grado de apalancamiento, la liquidez, el tamaño de la firma, la variabilidad en las ganancias y un coeficiente obtenido a partir de regresar las ganancias de la firma contra el promedio de las del mercado.

${ }^{13}$ Desde que el retorno es mayor en períodos de pagos de dividendos, de no incrementarse el riesgo habría oportunidades de beneficios lo cual sería de común conocimiento hacia todos los inversores.
} 
en la fusión y a la sensibilidad del ciclo de negocios de la economía ${ }^{14}$.

Brennan y Copeland (1988) estudiaron como pueden contribuir los split a la no estacionariedad de la distribución de los retornos de los activos, y así extendido a la inestabilidad de los betas. Encontraron que beta se incrementa cerca de un $20 \%$ luego del anuncio del split, aproximadamente un $30 \%$ cuando se hace efectivo, para luego situarse en un $18 \%$ por encima del beta pre-split.

Wells (1994) estimó los betas de unas diez acciones de la Bolsa de Estocolmo. Utilizando modelos de reversión a la media, de coeficientes aleatorios y de trayectoria al azar, además de permitir la posibilidad de cambio estructural utilizando el criterio de Akaike, concluyó que los betas son no estacionarios advirtiendo especial precaución en su uso. Señaló que, partiendo de la noción de beta, al expresar la correlación entre dos esperanzas matemáticas, no hay razón para que no cambie dado que las condiciones del mercado están lejos de ser estáticas. Es por ello que beta debe ser modelado como un proceso dinámico más que como un coeficiente constante en el tiempo.

Coutts, Roberts y Mills (1997) también estudiaron la estabilidad de los parámetros del MIS. Destacaron que al utilizar el modelo, a partir del uso de datos históricos, se requiere que los coeficientes sean estables no solamente en el período de estimación sino a futuro, donde se quiere aplicar dicha estimación. Si esto no es así, de poco sirve el poder predictivo del comovimiento pasado de la acción con el mercado. Usando datos del mercado londinense, y mediante una metodología de Mínimos Cuadrados Recursivos hallaron evidencia de la inestabilidad de los parámetros. Los autores atribuyeron este hecho a factores no inherentes a la firma (como la desregulación del mercado de valores de Londres y el crack en los mercados mundiales a fines de 1987), como a factores propios de la firma que no son capturados por el modelo.

Odabasi (2000) analizó la estacionariedad de beta aplicado a la Bolsa de Estambul, manifestando que para comparaciones multiperíodo es más adecuado mostrar la estacionariedad del ranking de betas más que su valor. No obstante, destaca la confiabilidad en el uso de betas por la relativa estacionariedad en portafolios de cinco o más acciones. Justamente, esto también es señalado por Fama y French (2004) en una revisión de trabajos empíricos sobre CAPM.

Grandes, Panigo y Pasquini (2006) muestran los patrones seguidos por el promedio de betas, en series de tiempo, por sectores económicos en países latinoamericanos. El patrón no estacionario encontrado, lo atribuyen a la baja liquidez en ciertas empresas, sectores y mercados. Concluyen que acciones con bajo volumen negociado (la mayoría en su muestra) están asociadas a betas volátiles debido a la gran cantidad de días en que no operan.

Por último, la no estacionariedad de beta puede calmar algunos debates en la literatura como los devenidos de los trabajos de Fama y French $(1992,1996 \mathrm{~b})$. Jaggannathan y Wang (1996) señalaron que el comportamiento inestable de beta en el tiempo es suficiente para revertir los resultados encontrados por Fama y French (1992). En particular, destacaron una versión condicional de CAPM,

\footnotetext{
${ }^{14}$ Las firmas adquiridas suelen pasar a tener un beta menor, mientras que las compradoras uno mayor.
} 
donde tanto beta como el premio por riesgo varian en el tiempo.

\section{Metodología de Estimación}

Como beta es presumiblemente no estacionaria en el tiempo, un plausible método de estimación es a través del Varying Coefficient Model (VCM), el cual es una generalización del Generalized Additive Model (GAM). En el GAM, en contraste con los modelos lineales, el regresando es modelado dependiendo aditivamente de manera suave aunque no especificada, de un número de variables explicativas.

El GAM tiene la siguiente forma:

$$
E\left(Y / X_{1}, X_{2}, \ldots ., X_{P}\right)=\alpha+f_{1}\left(X_{1}\right)+f_{2}\left(X_{2}\right)+\ldots .+f_{P}\left(X_{P}\right)=\alpha+\sum_{j=1}^{P} f_{j}\left(X_{j}\right)
$$

Donde $f_{j}$ representan las smooth functions, las cuales pueden ser estimadas por medio de cualquier $p$-dimensional scatterplot smoother $(f(X)=s(X))$, o bien asumiendo un modelo menos general: $s(X)=\sum_{j=1}^{P} s_{j}\left(X_{j}\right)$, estimando las $s_{j}$, de a una por vez iterativamente, usando un scatterplot smoother (Hastie y Tibshirani 1986). Adicionalmente es necesario asumir que $\sum_{i=1}^{N} f_{j}\left(X_{i j}\right)=0 \forall j$ para la identificación de los coeficientes ${ }^{15}$.

Un importante hecho de este procedimiento es que refina la maldición de la dimensionalidad (curse of dimentionality) inherente a varias técnicas no paramétricas tal como el estimador de Naradaya-Watson (Hastie, Tibshirani y Friedman 2001, Kauermann 2006 ${ }^{16}$.

El VCM es un modelo que es lineal en los regresores pero que sus coeficientes son permitidos variar suavemente con el valor de otras variables, denominadas Effect modifiers. En particular, sea $Y$ una variable aleatoria cuya distribución depende de un parámetro $\eta$, con regresores $X_{1}, X_{2}, \ldots ., X_{P} ; T_{1}, T_{2}, \ldots ., T_{P}$, el modelo toma la forma:

$$
E(Y / X, T)=g[m(X, T)]=g(\eta)=g\left[m_{0}(T)+X m_{X}(T)\right]
$$

\footnotetext{
${ }^{15}$ Este hecho va a hacer que las estimaciones de los coeficientes estén centradas en cero (ver gráfico 1)

${ }^{16}$ La maldición reside en la pérdida de convergencia del estimador no paramétrico al verdadero valor del parámetro cuando aumenta el número de regresores para el mismo tamaño de muestra (Pagan y Ullah 1999).
} 
Donde $g()$ es la función vínculo y $m(X, T)$ una función desconocida de suavizamiento (no paramétrica). Siguiendo a Kauermann (2006), se trata de una combinación de una escala métrica $(T)$ y regresores $(X s)$. Mientras que $m_{0}(T)$ es la influencia de $T, m_{X}(T)$ representa la interacción entre las variables $X$ y $T$, que de otra manera sería igual al coeficiente de regresión estándar que acompaña a la variable explicativa.

Alternativamente:

$$
\eta=\beta_{0}+X_{1} \beta_{1}\left(T_{1}\right)+X_{2} \beta_{2}\left(T_{2}\right)+\ldots .+X_{P} \beta_{P}\left(T_{P}\right)
$$

Las variables $T_{1}, T_{2}, \ldots ., T_{P}$ cambian los coeficientes de las $X_{1}, X_{2}, \ldots ., X_{P}$ a través de las funciones (no especificadas) $\beta_{1}(), \beta_{2}(), \ldots ., \beta_{P}()$.

La dependencia de las $\beta_{j}()$ sobre $T_{j}$ implica algún tipo de interacción entre cada $T_{j}$ y cada $X_{j}$ (Hastie y Tibshirani 1993). En el contexto de GAM, $\eta$ es una suma aditiva de smooth functions.

Para ser posible llevar adelante la estimación es necesario imponer restricciones sobre las funciones de los coeficientes de una u otra forma. De esta manera, el procedimiento propuesto se basa en Mínimos Cuadrados Penalizados (MCP) (Hastie y Tibshirani 1993). El suavizamiento de los términos es obtenido usando MCP con un suavizado de los parámetros seleccionado por medio de Generalised Cross-Validation (GCV), el cual es un método usado para elegir el grado de ajuste de una función. Dada una muestra de tamaño $N$, para la estimación de las $\beta_{j}()$ el algoritmo utilizado minimiza:

$$
\sum_{i=1}^{N}\left\{y_{i}-\sum_{j=1}^{P} x_{i j} \beta_{j}\left(t_{i j}\right)\right\}^{2}+\sum_{j=1}^{P} \lambda_{j} \int \beta_{j}^{\prime \prime}\left(t_{j}\right)^{2} d t_{j}
$$

Donde el primer término constituye una medida de la bondad de ajuste y el segundo penaliza la rigurosidad de cada $\beta_{j}$ con un parámetro fijo $\lambda_{j}$, el cual regula el trade-off entre suavizamiento y bondad de ajuste. Éste es óptimamente elegido por medio del algoritmo propuesto por Wood (2000). El MCP conlleva la misma esencia del estimador, más popular, spline smoothing (Eubank 1999, Pagan y Ullah 1999, Yatchew 2003).

Para testear la interacción entre la variable explicativa del MIS (el retorno del mercado) y el tiempo, proponemos estimar la siguiente ecuación:

$$
R s t=\alpha_{s}+\beta_{1 s} R_{M t}+\beta_{2 s}(t) R_{M t}+v_{s t} \quad t=1,2,3, \ldots ., T
$$

La cual es una adapatación del Dynamic Generalized Linear Model propuesto primaramente por West, Harrison y Migon (1985). Aquí las variables $T_{j}$ es solamente $t$ (tiempo), la cual es sospechada de tener efecto sobre el retorno del mercado $R_{M}$. Así, $\beta_{2 s}(t)$ es una función no paramétrica que captura el efecto 
del tiempo sobre el riesgo del sector $s$, mientras que $\beta_{1 s}$ es la media condicional y convencional del efecto del retorno del mercado sobre el retorno del sector en todo el período.

Una primera aplicación de esta metodología a la estimación de beta es aportada por Eisenbeiss, Kauermann y Semmler (2007) para la Bolsa Alemana durante 1991-2003 ${ }^{17}$. Ellos señalan que dependiendo del período en cuestión $(t=T)$ el valor de beta es aditivamente creciente o decreciente por la estimación de su no paramétrica contraparte: $\widehat{\beta}_{s T}=\widehat{\beta}_{1 s}+\widehat{\beta}_{2 s}(T)$.

Puede decirse que el modelo tiene carácter semiparamétrico en el sentido del efecto lineal de la variable explicativa sobre el regresando, mientras que el correspondiente coeficiente no es tratado como constante, sino como función del tiempo. Para cada valor de $t$, el modelo especifica una regresión de $R_{S}$ sobre $R_{M}$ con la pendiente de la función variando suavemente con $t$. En otras palabras, el modelo deja que beta se ajuste flexiblemente a los datos.

Adicionalmente, de manera de tratar por la posible fuente de heterocedasticidad en la varianza de los residuos, Eisenbeiss, Kauermann y Semmler (2007) sugieren estimar un Generalized Additive Gamma Model. Se trata de modelar la dependencia de la variación de los residuos con el tiempo de manera análoga al método de estimación en estudio.

\section{Datos y estadísticas preliminares}

\subsection{Base de datos}

La base de datos de este trabajo es producto de un exhaustivo proceso de recopilación de cotizaciones de cierre y volumen negociado de todas las empresas listadas en cada rueda en la BCBA. La base engloba la totalidad de las acciones que han cotizado durante el período 1994-2007.

Las empresas presentes en el período que han cotizado sus acciones fueron clasificadas en sectores según su actividad principal reportada en el Boletín Semanal de la BCBA $^{18}$.

Cada sector fue considerado como un portafolio de las acciones de las empresas que entran en su categoría, obteniéndose el retorno ponderado en cada sesión de rueda bursátil. La ponderación del retorno de cada empresa dentro del portafolio de su sector fue computado en base a su volumen negociado:

$$
R_{S T}=\sum_{i=1}^{n S} \theta_{i T} R_{i T}=\sum_{i=1}^{n S}\left(\frac{V_{i T}}{\sum_{i} V_{i T}} R_{i T}\right) \quad i=1,2, . ., n S
$$

\footnotetext{
${ }^{17}$ Desde ya aportan nueva evidencia acerca de la no estacionariedad de beta. ${ }^{18}$ Ver Anexo.
} 
Es decir, el retorno del sector ${ }_{S}$ en ${ }_{T}$ es igual a la suma ponderada $\left(\theta_{i t}\right)$, en base al volumen operado de la empresa $i\left(V_{i T}\right)$ en el volumen operado total del sector $\left(\sum_{i=1}^{n S} V_{i T}=V_{S T}\right)$, de los retornos de las firmas individuales $\left(R_{i T}\right)$.

Los portafolios sectoriales comparten ciclos de negocios, costos de insumos productivos, restricciones comerciales, carga impositiva, etc, pero esto no quiere decir que el agrupamiento sea homogéneo. Roll (1988) encuentra que, generalmente, grandes empresas tienden a tener portafolios más diversificados que las chicas. Bour (2007) muestra que sectores aparentemente homogéneos pueden presentar importantes diferencias en sus fundamentales.

Así también, para computar el retorno del mercado, en vez de usar los índices convencionales como buena aproximación aunque no exahustiva del movimiento global del mercado, se construyó un value-weighted index acorde a Brown y Warner (1980). Para ello computamos el portafolio de mercado en base a la siguiente ecuación:

$$
R_{M T}=\sum_{S=1}^{11} \pi_{s T} R_{s T}=\sum_{s=1}^{11}\left(\frac{V_{s T}}{\sum_{s} V_{s T}} R_{s T}\right) \quad s=1,2,3, . ., 11
$$

Es decir, el retorno del mercado en $T_{T}$ es igual a la suma ponderada $\left(\pi_{s t}\right)$, en base al volumen negociado del sector $s\left(V_{s T}\right)$ en el total negociado del mercado $\left(\sum_{s=1}^{11} V_{s T}=V_{M T}\right)$, de los retornos de los sectores $\left(R_{s T}\right)$.

Es importante notar que tanto los portafolios de sectores como el del mercado global, fueron rebaleanceados diariamente de acuerdo al volumen operado.

Debido a que durante el período se han producido splits, canjes de acciones y variación de capital social, así como hubo empresas con muy baja liquidez, todos estos hechos posiblemente hicieron que los valores de mercado de las acciones hayan variado fuertemente entre un día y otro, distorcionando el retorno real de mercado. Ante la alternativa poco práctica de corregir cada valor manualmente, una alternativa estadística consiste en dejar de lado los valores atípicos (outliers) con un nivel de significatividad de 0,01. De esta manera, los datos usados en este trabajo aislan los retornos debido a estos fenómenos.

\subsection{Consideraciones adicionales de los datos utilizados}

Una cuestión adicional reside en la naturaleza misma de los datos. Si bien algunas de las caracteríticas que exiben las distribuciones de retornos de acciones, más aún en mercados emergentes (Wong Dávila 2003), pueden no ser problemáticas por la flexibilidad del modelo, como la no normalidad de la distribución (Kauermann 2006), otras características pueden ser problemáticas. Aunque el CAPM esté limitado a un análisis de media y varianza, sin embargo la asímetría y la curtosis son más aparentes en las distribuciones de retornos de acciones de mercados emergentes, lo que sugiere un mayor riesgo a la baja 
(downside risk). Por ello, Galagedera (2006) aconseja trabajar con downside beta, ligado al concepto de semivarianza, en mercados emergentes debido a que las distribuciones de los retornos presentan mayor dispersión, asimetría y curtosis. No obstante, el objetivo de este trabajo es testear la estabilidad de la medida convencional de riesgo sistemático, con lo que se utilizará beta y no el downside beta.

Campbell (2001) señala que uno de los supuestos del modelo es la ausencia de costos de transacción, de manera que el arbitraje entre activos pueda actuar perfectamente. En Argentina, los costos de transacción relacionados a la operativa con acciones son más elevados que los presentes en las bolsas de países desarrollados. Por otro lado, la segmentación de mercado, debido a los costos asociados a comprar y vender activos financieros fuera del país, no trae problemas dado que el estudio se basa solamente en el mercado local.

Campbell (2001) también apunta que en los mercados emergentes hay pocas firmas por sectores y menos sectores económicos cotizando que en los mercados desarrollados. Adicionalmente, muchas de las firmas suelen tener concentrados sus negocios en el mercado interno, con lo que sus negocios tienden a moverse con el ciclo económico. En nuestra muestra, ocurre de hecho la primera observación y presumiblemente la segunda.

En otro rango de cosas, Grandes, Panigo y Pasquini (2006) destacan que la iliquidez de las acciones latinoamericanas y la incertidumbre macroeconómica pueden resultar en una alta volatilidad de beta ${ }^{19}$. Justamente, Ferraro (2007) y Olivieri y Ferraro (2007) muestran varias medidas de liquidez para los mercados accionarios latinoamericanos, evidenciando una relativa y absoluta baja liquidez de la BCBA sumada a una caída de la misma en los últimos años ${ }^{20}$. No obstante, al trabajar con portafolio de sectores, buena parte de la iliquidez se deja de lado.

\subsection{Estadísticas Preliminares}

El siguiente cuadro muestra por sector económico la cantidad de empresas, el volumen negociado y el porcentaje que cada uno representa en el total negociado para el período en su conjunto.

\footnotetext{
${ }^{19}$ También advierten sobre los cambios en los índices bursátiles debido a listado y delistado de firmas, fusiones y adquisiciones, etc. No obstante aquí, como fue expuesto, el portafolio de mercado no fue tomado a partir de índices bursátiles sino construído a partir de la totalidad del mercado accionario.

${ }^{20} \mathrm{El}$ volumen negociado en acciones respecto al PBI en la BCBA cayó $17 \%$ entre 2000 y 2007. Además, el mercado local fue el de más baja negociación en términos absolutos durante el primer semestre de 2007, dentro de un conjunto formado por las bolsas de Brasil, México, Chile y Perú.
} 
Cuadro 1: Cantidad de empresas, volumen negociado y porcentaje del volumen negociado según sectores económicos (1994-2007).

\begin{tabular}{cccc}
\hline \hline Sector & $\begin{array}{c}\text { Cantidad de } \\
\text { Empresas }\end{array}$ & $\begin{array}{c}\text { Volumen } \\
\text { (millones de pesos) }\end{array}$ & $\begin{array}{c}\text { Part. en el } \\
\text { Vol. Mercado }\end{array}$ \\
\hline \hline Alimenticio & 15 & 2706.5 & $2.4 \%$ \\
\hline Comercial & 11 & 266.1 & $0.2 \%$ \\
\hline Construcción & 9 & 1306.3 & $1.2 \%$ \\
\hline Eléctrico & 9 & 3433.6 & $3.1 \%$ \\
\hline Financiero & 22 & 25504.5 & $23.1 \%$ \\
\hline Holding & 5 & 7108.6 & $6.4 \%$ \\
\hline Industrial & 39 & 5308.2 & $4.8 \%$ \\
\hline Metalúrgico & 8 & 29313.5 & $26.5 \%$ \\
\hline Otros & 17 & 1133.2 & $1.0 \%$ \\
\hline Petrolero & 21 & 23446.3 & $21.2 \%$ \\
\hline Telecomunicaciones & 4 & 11091.5 & $10.0 \%$ \\
\hline
\end{tabular}

Fuente: Elaboración propia en base a Economática y BCBA.

Si bien el sector con mayor presencia de empresas es el industrial, no llega a representar el $5 \%$ del volumen negociado durante el período. En oposición, el sector metalúrgico negoció más del $26 \%$ con apenas 8 empresas. El sector comercial y los otros sectores suman 28 empresas pero apenas cuentan por el $1,2 \%$ del total negociado. El corolario de estos hechos, es la alta concentración de la negociación en ciertos sectores (metalúrgico, financiero y petrolero). Estos tres sectores contaron por el $71 \%$ del volumen operado con el $32 \%$ de las empresas de la muestra.

El cuadro 2 muestra el retorno promedio diario, el desvío estándar y el retorno por unidad de desvío estándar para cada uno de los sectores económicos.

Cuadro 2: Retorno promedio diario, desvío estándar y retorno por unidad de desvío estándar según sectores económicos (1994-2007).

\begin{tabular}{cccc}
\hline \hline & Retorno pmedio(1) & Desvío estándar(2) & $(1) /(2)$ \\
\hline \hline Alimenticio & 0.054 & 2.445 & 0.022 \\
\hline Comercial & 0.153 & 2.251 & 0.068 \\
\hline Construcción & 0.134 & 2.493 & 0.054 \\
\hline Eléctrico & 0.037 & 2.272 & 0.016 \\
\hline Financiero & 0.106 & 2.555 & 0.042 \\
\hline Holding & 0.179 & 2.620 & 0.068 \\
\hline Industrial & 0.210 & 2.797 & 0.075 \\
\hline Metalúrgico & 0.149 & 2.511 & 0.059 \\
\hline Otros & 0.051 & 2.459 & 0.021 \\
\hline Petrolero & 0.132 & 2.038 & 0.065 \\
\hline Telecomunicaciones & 0.019 & 2.494 & 0.008 \\
\hline \hline
\end{tabular}

Fuente: Elaboración propia en base a Economática y BCBA. 
El sector que arrojó mayor retorno promedio diario, durante el período, fue el industrial, seguido por holding. Además, al corregir por riesgo, también el sector industrial fue el que observó el mayor retorno por unidad de riesgo asumida. No obstante, fue el que evidenció mayor dispersión en sus retornos diarios. En cambio, el sector petrolero fue el que presentó menor dispersión en sus retornos, seguido por el comercial y el eléctrico. Por último, los sectores que menos rindieron, en relación a su riesgo, fueron el de telecomunicaciones, el eléctrico, otros sectores y el alimenticio.

El cuadro 3 indaga la cantidad de días en que el retorno de cada sector superó el $5 \%$, como así también el rango entre el retorno máximo y el mínimo, y los retornos correspondientes a los percentiles 1 y 99 .

Cuadro 3: Cantidad de días con retorno superior al $5 \%$, rango de retornos y retornos correspondientes a los percentiles 1 y 99 según sectores económicos (1994-2007)

\begin{tabular}{ccccc}
\hline \hline Sector & Días Ret. $>5 \%$ & Rango & P1 & P99 \\
\hline \hline Alimenticio & 86 & 20.989 & -6.920 & 6.857 \\
\hline Comercial & 102 & 23.947 & -6.818 & 7.692 \\
\hline Construcción & 110 & 24.581 & -6.564 & 7.024 \\
\hline Eléctrico & 77 & 20.014 & -6.202 & 6.375 \\
\hline Financiero & 103 & 24.440 & -7.046 & 7.288 \\
\hline Holding & 122 & 27.267 & -6.863 & 8.003 \\
\hline Industrial & 143 & 26.382 & -7.275 & 8.044 \\
\hline Metalúrgico & 91 & 22.018 & -6.354 & 6.634 \\
\hline Otros & 96 & 27.421 & -6.790 & 7.078 \\
\hline Petrolero & 50 & 18.851 & -5.579 & 5.429 \\
\hline Telecomunicaciones & 89 & 23.979 & -6.521 & 6.697 \\
\hline
\end{tabular}

Fuente: Elaboración propia en base a Economática y BCBA.

El sector industrial fue aquel que tuvo mayor cantidad de días en donde el retorno diario superó el $5 \%$, además de mostrar el tercer mayor rango de retornos, luego de otros sectores y holding. En tanto, el sector petrolero fue el que menos cayó en un día, a juzgar por el $99 \%$ de sus retornos que menos cayeron. En adición, también fue el portafolio de acciones que mostró menor rango de retornos.

Es también interesante observar la correlación entre los retornos de los distintos sectores (cuadro 4). 


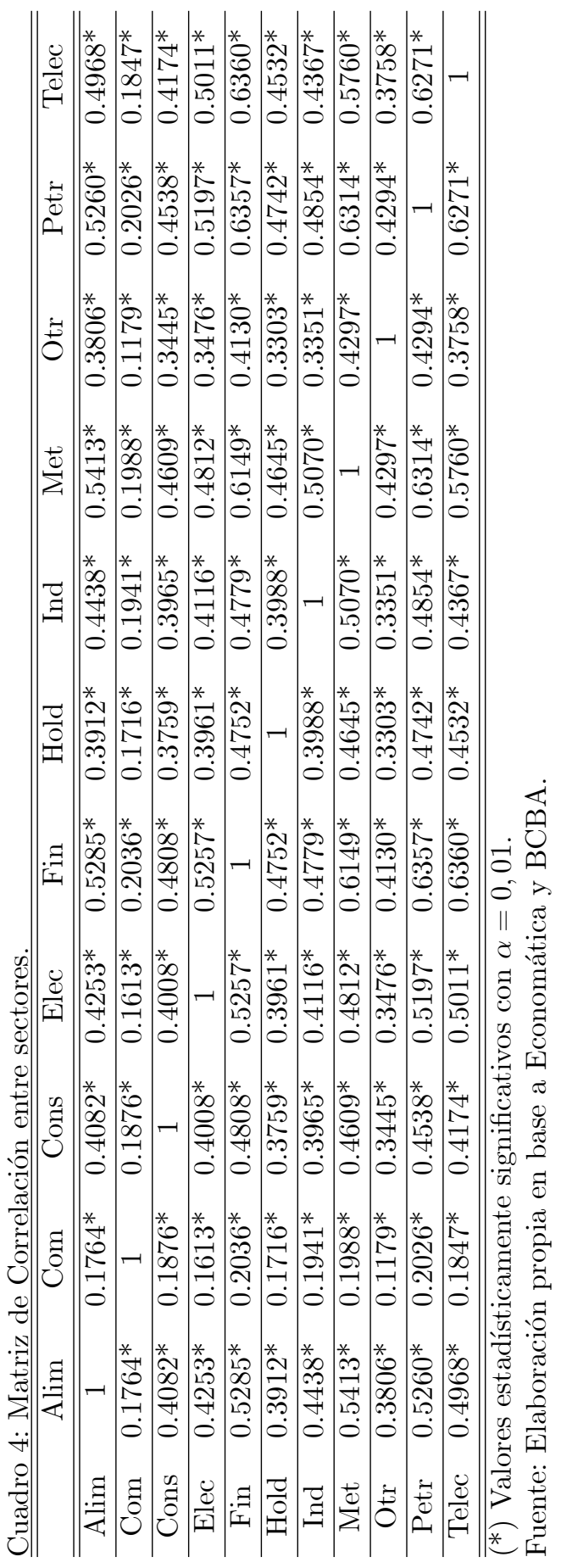


Los sectores que estuvieron más correlacionados fueron telecomunicaciones con financiero, petrolero con financiero, metalúrgico con petrolero, y telecomunicaciones con petrolero. En tanto, los sectores menos correlacionados fueron comercial y otros sectores, eléctrico y comercial, y holding con comercial. Justamente, el sector comercial muestra las menores correlaciones.

\section{Resultados}

El software R, de dominio público, constituye un excelente recurso computacional para correr este tipo de modelos. Los resultados de la estimación por MCO, considerando todo el período en cuestión, son mostrados en el cuadro 5. Los resultados de la estimación del VCM aparecen en el cuadro 6 y en el gráfico 1.

Cuadro 5: Estimación de betas, desvío estándar y bondad de ajuste de la estimación por $\mathrm{MCO}$

\begin{tabular}{rrrr}
\hline \hline Sector & Intercepto & Beta & R2AJ.MCO \\
\hline \hline & $(0.03366)$ & $(0.01632)$ & 0.404 \\
\hline Alimenticio & $-0.08784^{* *}$ & $0.75703^{* * *}$ & \\
\hline & $0.10316^{* *}$ & $0.26497^{* * *}$ & 0.058 \\
\hline Comercial & $(0.03896)$ & $(0.01890)$ & \\
\hline Construcción & 0.008038 & $0.675024^{* * *}$ & 0.309 \\
\hline & $(0.036948)$ & $(0.017919)$ & \\
\hline Eléctrico & $-0.09037^{* *}$ & $0.68081^{* * *}$ & 0.378 \\
\hline & $(0.03195)$ & $(0.01549)$ & \\
\hline Financiero & $-0.08788^{* * *}$ & $1.03642^{* * *}$ & 0.694 \\
\hline & $(0.02520)$ & $(0.01222)$ & \\
\hline Holding & 0.03516 & $0.76925^{* * *}$ & 0.363 \\
\hline & $(0.03729)$ & $(0.01808)$ & \\
\hline Industrial & 0.05496 & $0.83100^{* * *}$ & 0.372 \\
\hline & $(0.03953)$ & $(0.01917)$ & \\
\hline Metalúrgico & -0.04014 & $1.00838^{* * *}$ & 0.680 \\
\hline Otros & $(0.02532)$ & $(0.01228)$ & \\
\hline & -0.05845 & $0.58711^{* * *}$ & 0.240 \\
\hline Petrolero & $(0.03822)$ & $(0.01854)$ & \\
\hline & -0.023155 & $0.828005^{* * *}$ & 0.696 \\
\hline Telecomunicaciones & $-0.020050)$ & $(0.009724)$ & \\
\hline \hline & $\left(0.027802^{* * *}\right.$ & $0.94728^{* * *}$ & 0.608 \\
\hline \hline
\end{tabular}

(***) Estadíticamente significativa con $\alpha=0.0001$

(**) Estadíticamente significativa con $\alpha=0.001$

(*) Estadíticamente significativa con $\alpha=0.01$ 
Cuadro 6: Estimación de la parte paramétrica de betas, desvío estándar y bondad de ajuste en la estimación por VCM.

\begin{tabular}{|c|c|c|c|}
\hline Sector & Intercepto & Beta & R2AJ.VCM \\
\hline \multirow[t]{2}{*}{ Alimenticio } & $-0.12995^{* * *}$ & $0.74124^{* * *}$ & 0.476 \\
\hline & $(0.03162)$ & $(0.01594)$ & \\
\hline \multirow[t]{2}{*}{ Comercial } & 0.05966 & $0.32609^{* * *}$ & 0.0826 \\
\hline & $(0.04179)$ & $(0.02071)$ & \\
\hline \multirow[t]{2}{*}{ Construcción } & -0.01858 & $0.70487 * * *$ & 0.393 \\
\hline & $(0.03634)$ & $(0.01833)$ & \\
\hline \multirow[t]{2}{*}{ Eléctrico } & $-0.06704^{\prime}$ & $0.44943^{* * *}$ & 0.175 \\
\hline & $(0.03822)$ & $(0.01937)$ & \\
\hline \multirow[t]{2}{*}{ Financiero } & $-0.09713^{* * *}$ & $1.00744^{* * *}$ & 0.393 \\
\hline & $(0.02307)$ & $(0.01191)$ & \\
\hline \multirow[t]{2}{*}{ Holding } & -0.006859 & $0.773017^{* * *}$ & 0.538 \\
\hline & $(0.033140)$ & $(0.019037)$ & \\
\hline \multirow[t]{2}{*}{ Industrial } & 0.02119 & $0.83077^{* * *}$ & 0.631 \\
\hline & $(0.03305)$ & $(0.01884)$ & \\
\hline \multirow[t]{2}{*}{ Metalúrgico } & -0.01603 & $0.83077^{* * *}$ & 0.81 \\
\hline & $(0.01921)$ & $(0.01884)$ & \\
\hline \multirow[t]{2}{*}{ Otros } & $-0.09533^{*}$ & $0.57275^{* * *}$ & 0.292 \\
\hline & $(0.03777)$ & $(0.01900)$ & \\
\hline \multirow[t]{2}{*}{ Petrolero } & -0.02201 & $0.82504^{* * *}$ & 0.701 \\
\hline & $(0.02154)$ & $(0.01091)$ & \\
\hline \multirow[t]{2}{*}{ Telecomunicaciones } & $-0.14999^{* * *}$ & $0.93301^{* * *}$ & 0.661 \\
\hline & $(0.02660)$ & $(0.01362)$ & \\
\hline
\end{tabular}

(***) Estadíticamente significativa con $\alpha=0.0001$

(**) Estadíticamente significativa con $\alpha=0.001$

$\left(^{*}\right)$ Estadíticamente significativa con $\alpha=0.01$

(') Estadíticamente significativa con $\alpha=0.05$ 


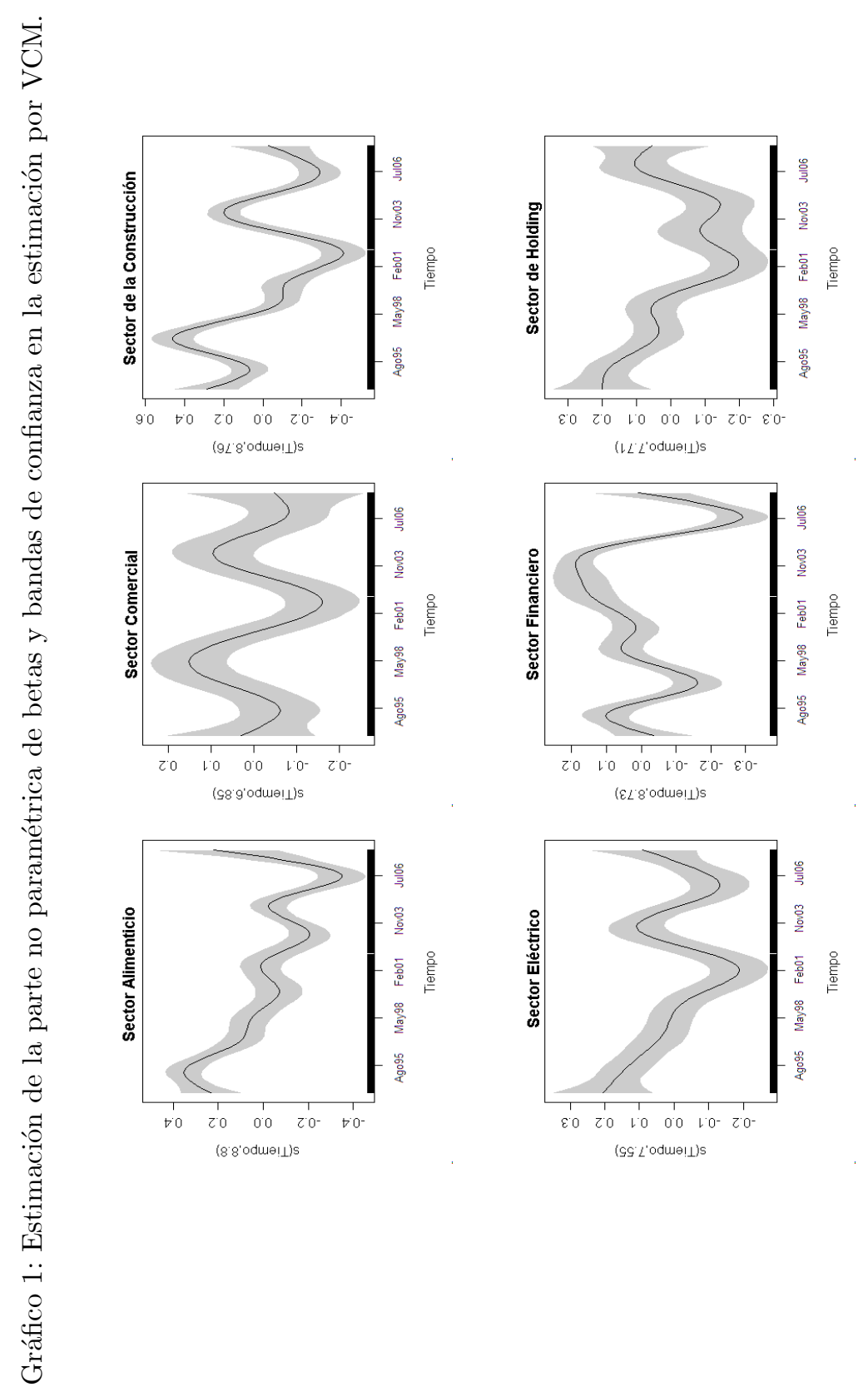



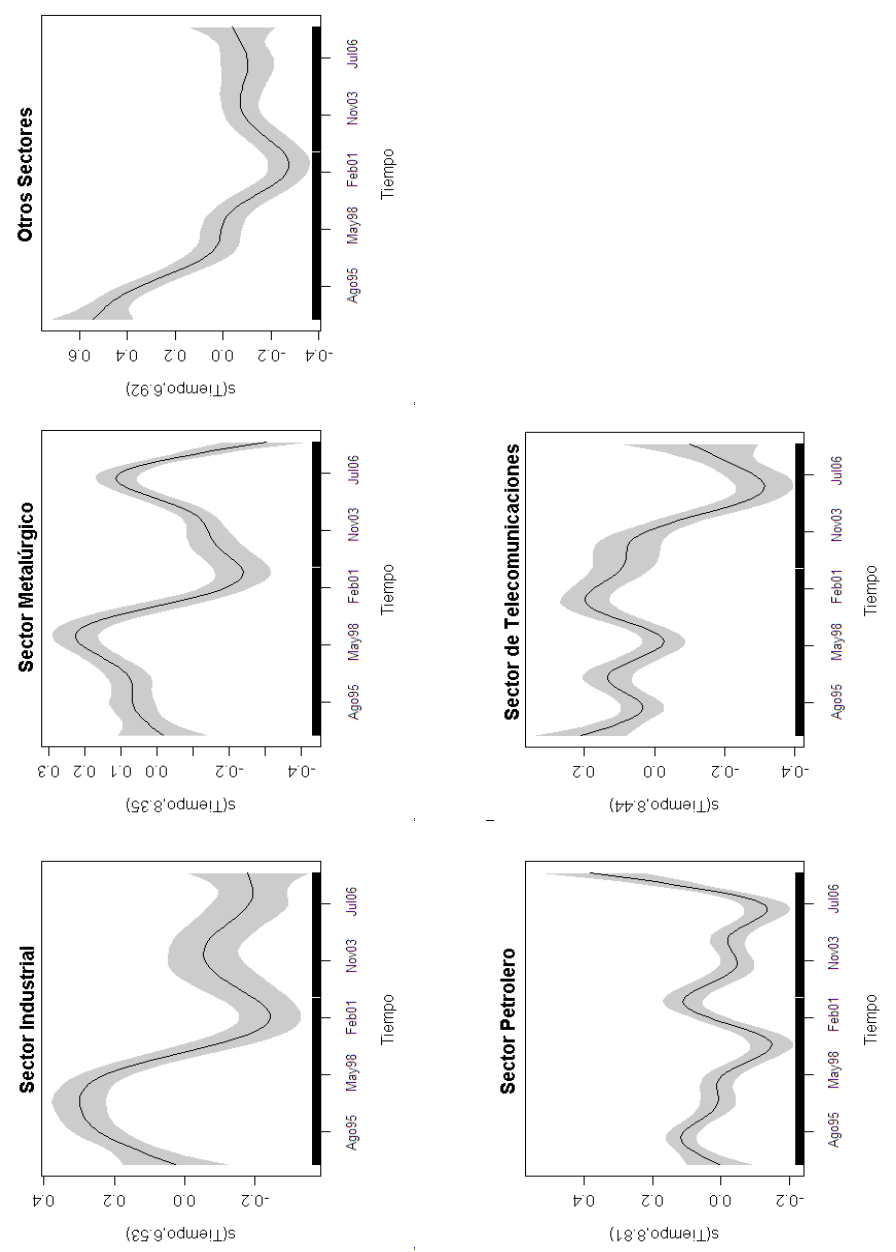
Estimando el MIS por MCO, observamos que todos los coeficientes beta, no así su intercepto, son estadísticamente significativos, con lo que el mercado es un adecuado regresor para explicar los movimientos en los retornos del portafolio de los sectores. La estimación muestra al sector financiero y al metalúrgico con beta mayor a uno. En cambio, los sectores comercial y otros sectores han sido los sectores con menor riesgo sistemático. En otras palabras, los que menos reaccionaron ante movimientos del mercado. No obstante, el $R^{2}$ ajustado, por construcción, señala que los retornos de los sectores metalúrgico, petrolero y financiero fueron los mejores explicados por el mercado.

Sin embargo, tal como mencionamos más arriba, estos coeficientes no pueden ser útiles si no son estables en el tiempo. Como muestra la estimación por VCM en el gráfico 1, el beta de cada sector se desvía significativamente respecto a su valor medio, presentado en el cuadro 6 , centrado en cero como condición necesaria de los modelos GAM con el propósito de identificación. Todos los sectores considerados muestran un patrón volátil de su beta en el tiempo. En particular el sector comercial se mostró como el de beta más variable. El único sector que evidenció beta estable durante algún período de tiempo, fueron los otros sectores en los últimos tres años de la muestra.

Por otro lado, todos los sectores, con excepción del metalúrgico y holding, evidenciaron una caída en el valor de su beta en los años que siguieron a la crisis financiera de 2001/02 hasta cuando comenzó a cambiar el contexto internacional, primeramente con el derrumbe del mercado accionario chino y luego con la crisis de hipotecas de alto riesgo.

Es interesante observar la dinámica seguida por el beta del sector financiero. Durante la crisis de 2001/02 su beta mostró los valores más altos del período para luego caer fuertemente con la recuperación del sector. Sin embargo, la tendencia cambió cuando comenzó la mayor volatilidad en los mercados internacionales para luego aumentar fuertemente desatada la crisis de crédito a nivel mundial. De hecho, dicha crisis propició un aumento en el riesgo sistemático en cada uno de los sectores excepto para el metalúrgico y holding. Por ejemplo, el sector alimenticio venía mostrando, aunque de manera escalonada, una caída en el valor de su beta para luego aumentar abruptamente durante el último año de la muestra.

En suma, hay suficiente evidencia de que el término de interacción entre el retorno de mercado y el tiempo es necesario en la especificación del modelo. Incluso hay que tener en cuenta que de trabajar directamente con las acciones y no con portafolios, el patrón de beta sería aún más variable tal como advierten Klemkosky y Martin (1975), Odabasi (2000) y Fama y French (2004).

A fin de relacionar los patrones de betas de los sectores económicos con el propio mercado, el gráfico 2 muestra el retorno del mercado en el tiempo. 
Gráfico 2: Sendero suavizado de los retornos del portafolio del mercado

(1994-2007).

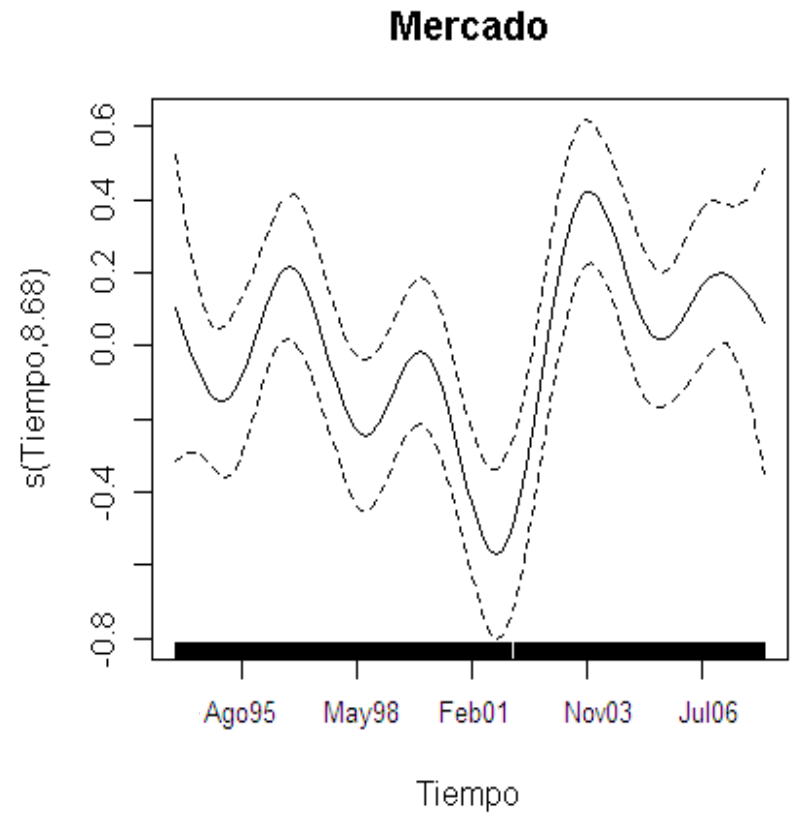

Como se observa, el gráfico captura la caída tras las crisis en los mercados internacionales (México 1994, Sudeste de Asía 1997, Rusia 1998), el desplome hacia la crisis de 2001/02 junto a la consecuente recuperación desde entonces, y los efectos de la crisis de crédito internacional al final del período. Como apuntan Eisenbeiss, Kauermann y Semmler (2007), los inversores posicionados en sectores que muestran un sendero de su beta que se mueve en forma contracíclica con el retorno del mercado, exigen mayor premio por riesgo en épocas de crisis. En nuestro caso, en el año previo a la crisis financiera de 2001/02, los sectores alimenticio, telecomunicaciones y petrolero fueron los que observaron un incremento en su riesgo. Desde el estallido de la crisis de hipotecas de alto riesgo, todos los inversores posicionados en cualquiera de los sectores, con excepción del metalúrgico, exigieron un mayor premio por el riesgo que asumen. 


\section{Conclusiones}

El concepto de beta constituye uno de los más importantes en el campo de las finanzas. Incluso podría decirse que es uno de los coeficientes que más interés despierta entre los agentes debido a que es utilizado en múltiples aplicaciones de la vida financiera cotidiana. Como el riesgo individual de los activos puede reducirse mediante diversificación, el beta del activo constituye una buena aproximación como medida de riesgo. Sin embargo, su utilidad requiere la factibilidad de su estimación. En la práctica, los agentes utilizan datos históricos para su estimación y extrapolan este valor a futuro, asumiendo estabilidad en el tiempo.

En las últimas décadas se han sucedido toda una batería de trabajos en el mundo académico cuestionando la estabilidad intertemporal de beta. Si bien los primeros trabajos se han basado en metodologías simples, el mayor desarrollo de modelos, algoritmos y sistemas computacionales han permitido testear mediante técnicas más sofisticadas.

Este trabajo constituye un aporte a la literatura principalmente en dos sentidos. Por un lado, aporta nueva evidencia sobre la inestabilidad de betas en portafolio de acciones. Por otro lado, constituye uno de los pocos trabajos académicos aplicados al mercado accionario argentino. En este sentido, aporta evidencia sobre el patrón seguido por los betas de portafolios de sectores económicos con oferta pública.

El trabajo revela que durante el período bajo análisis, ningún sector mostró un comportamiento estable de su beta. Es por ello que es aconsejable tener especial cuidado en utilizar betas históricas de aquellos sectores que han mostrado un sendero altamente volátil (e.j. sector comercial). Adicionalmente, hay una clara evidencia de que el riesgo sistemático de la mayoría de los sectores ha caído luego de la crisis financiera de 2001/02, junto con la recuperación económica, aunque gran parte de esa caída se haya evaporado con la mayor volatilidad internacional presente en el último año de la muestra.

No obstante, el modelo utilizado deja mucho del movimiento de los retornos de los sectores por explicar, a juzgar por el valor de los $R^{2}$ ajustado, aunque gana en el patrón de ajuste. Es por ello, que una línea de investigación a futuro debe indagar la conveniencia de introducir más factores como explicativos pero dejando librada la especificación lineal para introducir un mejor ajuste de los datos. 


\section{Referencias}

- Alexander, G. y Chervany, N. (1980). 'On the Estimation and Stability of Beta'. The Journal of Financial and Quantitative Analysis, Vol. 15, No. 1, pp. 123-137.

- Baesel, J. (1974). 'On the Assessment of Risk: Some Further Considerations'. The Journal of Finance, Vol. 29, No. 5, pp. 1491-1494.

- Bandi, F. y Perron, B. (2008). 'Long-run Risk-return Trade-offs'. Journal of Econometrics, Vol. 143, pp. 349-374.

- Banz, R. (1981). 'The relationship between return and market value of common stocks'. Journal of Financial Economics, Vol. 9, No. 1, pp. 3-18.

- Basu, S. (1977). 'Investment Performance of Common Stocks in Relation to Their Price-Earnings Ratios: A Test of the Efficient Market Hypothesis'. The Journal of Finance, Vol. 32, No. 3, pp. 663-682.

- Bawa, V. (1975). 'Optimal Rules for Ordering Uncertain Prospects'. Journal of Financial Economics, Vol. 2, Issue 1, pp. 95-121.

- Bawa, V. y Lindenberg, E. (1977). 'Capital Market Equilibrium in a MeanLower Partial Moment Framework'. Journal of Financial Economic, Vol. 5, Issue 2, pp. 189-200.

- Beaver, W., Kettler, P. y Scholes, M. (1970). 'The Association Between Market Determined and Accounting Determined Risk Measures'. The Accounting Review, Vol. 45, pp. 654-682.

- Beaver, W. y Manegold, J. (1975). 'The Association Between MarketDetermined and Accounting-Determined Measures of Systematic Risk: Some Further Evidence'. The Journal of Financial and Quantitative Analysis, Vol. 10, No. 2, pp. 231-284.

- Bhandari, L. (1988). 'Debt/Equity Ratio and Expected Common Stock Returns: Empirical Evidence'. The Journal of Finance, Vol. 43, No. 2, pp. 507-528.

- Black, F. (1972). 'Capital Market Equilibrium with Restricted Borrowing'.The Journal of Business, Vol. 45, No. 3, pp. 444-455.

- Blume, M. (1971). 'On the Assessment of Risk'. The Journal of Finance, Vol. 26, No. 1, pp. 1-10.

- Blume, M. (1975). 'Betas and their Regression Tendencies'. The Journal of Finance, Vol. 30, No. 3, pp. 785-795. 
- Bos, T. y Newbold, P. (1984). 'An Empirical Investigation of the Possibility of Stochastic Systematic Risk in the Market Model'. The Journal of Business, Vol. 57, No. 1, Part 1, pp. 35-41.

- Bour, A. (2007). 'Comentario sobre Un Estudio No Paramétrico sobre la Estabilidad de los Coeficientes Beta en Sectores con Oferta Pública de Acciones Locales'. XLII Reunión Anual de la AAEP.

- Brealey, R., Myers, S. y Marcus, A. (2001) 'Fundamentals of Corporate Finance'. Third Edition. Mcgraw Hill.

- Breen, W. y Lerner, E. (1973). 'Corporate Financial Strategies and Market Measures of Risk and Return'. The Journal of Finance, Vol. 28, No. 2, pp. 339-351.

- Brennan, M. y Copeland, T. (1988). 'Beta Changes Around Stock Splits: A Note'. The Journal of Finance, Vol. 43, No. 4, pp. 1009-1013.

- Brigham, E. y Houston, J. (2005). "Fundamentos de la Administración Financiera". Décima Edición. Thomson Ed. México.

- Brown, S. y Warner, J. (1980). 'Measuring Security Price Performance'. Journal of Financial Economics. Vol 8, pp 205258.

- Campbell, H. (2001). 'Asset Pricing in Emerging Markets'. National Bureau of Economic Research.

- Campbell, J., Lo, A. y MacKinlay, A. (1997). 'The Econometrics of Financial Markets'. Princeton University Press.

- Chen, S. (1981). 'Beta Nonstationarity, Portfolio Residual Risk and Diversification'. The Journal of Financial and Quantitative Analysis, Vol. 16, No. 1, pp. 95-111.

- Citigroup (2007). Çompany in-Deph: Edenor SA". Citigroup Global Markets, Equity Research.

- Conn, R. (1985). 'A Re-Examination of Merger Studies that Use the Capital Asset Pricing Model Methodology'. Cambridge Journal of Economics, Vol. 9, pp. 43-56.

- Coutts, J., Roberts, J. y Mills, T. (1997). 'Parameter Stability in the Market Model: Tests and Time Varying Parameter Estimation With UK Data'. The Statistician, Vol. 46, No. 1, pp. 57-70.

- Eisenbeiss, M., Kauermann, G. y Semmler, W. (2007). 'Estimating BetaCoefficients of German Stock Data: A Non-Parametric Approach'. Universität Bielefeld.

- Elton, E. y Gruber, M. (1995). 'Modern Portfolio Theory and Investment Analysis'. Fifth Edition. John Wiley \& Sons, Inc. 
- Eubank, R. (1999). 'Nonparametric Regression and Spline Smoothing' Second Edition. Marcel Dekker, Inc.

- Fabozzi, F. y Francis, J. (1977). 'Stability Tests for Alphas and Betas Over Bull and Bear Market Conditions'. The Journal of Finance, Vol. 32, No. 4, pp. 1093-1099.

- Fabozzi, F. y Francis, J. (1978). 'Beta as a Random Coefficient'. The Journal of Financial and Quantitative Analysis, Vol. 13, No. 1, pp. 101116 .

- Fama, E. y French, K. (1992). 'The Cross-Section of Expected Stock Returns'. Journal of Finance, Vol 47, No. 2, pp. 427-465.

- Fama, E. y French, K. (1996a). 'Multifactor Explanations of Asset Pricing Anomalies'. The Journal of Finance, Vol. 51, No. 1, pp. 55-84.

- Fama, E. y French, K. (1996b). 'The CAPM is Wanted, Dead or Alive'. The Journal of Finance, Vol. 51, No. 5, pp. 1947-1958.

- Fama, E. y French, K. (2004). 'The Capital Asset Pricing Model: Theory and Evidence'. The Journal of Economic Perspectives, Vol. 18, No. 3, pp. $25-46$.

- Fama, E. y MacBeth, J. (1973). 'Risk, Return, and Equilibrium: Empirical Tests'. Journal of Political Economy, Vol. 81, No. 3, pp. 607-636.

- Galagedera, D. (2006). 'An Alternative Perspective on the Relationship between Dowside Beta and CAPM Beta'. Emerging Markets Review, Vol. 8, pp. 4-19.

- Ferraro, M. (2007). 'Caracteríticas del Mercado de Capitales en Arhentina: Una Visión Comparativa'. Documento de Trabajo No. 18. Centro para la Estabilidad Financiera.

- Grandes, M., Panigo, D. y Pasquini, R. (2006). 'The Cost of Equity in Latin America'. Working Paper No. 12. Center for Financial Stability.

- Gonedes, N. (1973). 'Evidence on the Infoormation Content of Accounting Numbers: Accounting-based and Market-based Estimates of Systematic Risk'. The Journal of Financial and Quantitative Analysis, Vol. 8, No. 3, pp. 407-443.

- Hastie, T. y Tibshirani, R. (1986). 'Generalized Additive Models'. Statiscal Science, Vol. 1, No. 3. pp. 297-310.

- Hastie, T. y Tibshirani, R. (1993). 'Varying-Coefficient Models'. Journal of the Royal Statistical Society. Series B (Methodological), Vol. 55, No. 4, pp.757-796. 
- Hastie, T., Tibshirani, R. y Friedman, J. (2001). 'The Elements of Statiscal Learning: Data Mining, Inference and Prediction'. Springer.

- Hogar, W. y Warren, J. (1974) 'Toward the Development of an Equilibrium Capital Market Model Based on Semivariance'. Journal of Financial and Quantitative Analysis, Vol. 9, No. 1, pp. 1-11.

- Jagannathan, R. y Wang, Z. (1996). 'The Conditional CAPM and the Cross-Section of Expected Returns'. The Journal of Finance, Vol. 51, No. 1, pp. 3-53.

- Kalay, A. y Loewenstein, U. (1985). 'Predictable Events and Excess Returns: The Case of Dividend Announcements'. Journal of Financial Economics, Vol. 14, pp. 423-449.

- Kauermann, G. (2006). 'Nonparametric Models and their Estimation'. Archiv 90, 137-152. Allgemeiness Statistisches.

- Klemkosky, R. y Martin, J. (1975). 'The Adjustment of Beta Forecasts'. The Journal of Finance, Vol. 30, No. 4, pp. 1123-1128.

- Kryzanowski, L. y To, M. (1984). 'The Telescopic Effect of Past Return Realizations on ex-post Beta Estimates'. The Financial Review, Vol. 19, Issue 1, pp 1-25.

- Levy, R. (1971). 'Stationarity of Beta Coefficients' Financial Analysts Journal, Vol. 27, pp. 55-62.

- Levy, R. (1974). 'Beta Coefficients as Predictors of Return'. Financial Analysts Journal, Vol. 30, No. 1, pp. 61-69

- Lintner, J. (1965). 'The Valuation of Risk Assets and the Selection of Risky Investments in Stock Portfolios and Capital Budgets'. The Review of Economics and Statistics, Vol. 47, No. 1, pp. 13-37.

- Markowitz, H. (1952). 'Portfolio Selection'. The Journal of Finance, Vol. 7, No. 1, pp.77-91.

- Melicher, R. (1974). 'Financial Factors wich Influence Beta Variations within an Homogeneous Industry Enviroment'. The Journal of Financial and Quantitative Analysis, Vol. 9, No. 2, pp. 231-241.

- Mossin, J. (1966). 'Equilibrium in a Capital Asset Market'. Econometrica, Vol. 34, No. 4, pp. 768-783.

- Odabasi, A. (2000). 'Evidence on the Stationarity of Beta Coefficients: The Case of Turkey'. Bogazici University, Bebek, Istanbul 80815, Turkey.

- Olivieri, C. y Ferraro, M. (2007). 'Características del Mercado de Capitales en Argentina'. Fundación Bolsa de Comercio de Buenos Aires. 
- Pagan, A. y Ullah, A. (1999). 'Non parametric Econometrics'. Cambridge University Press.

- Pedersen, C. y Hwang, S. (2007). 'Does Downside Beta Matter in Asset Pricing?'. Applied Financial Economics, Vol. 17, Issue 12, pp. 961-978.

- Pennacchi, G. (2007). 'Theory of Asset Pricing'. First Edition. Addison Wesley.

- Price, K., Price, B. y Nantell, T. (1982). 'Variance and Lower Partial Moment Measures of Systematic Risk: Some Analytical and Empirical Results'. The Journal of Finance, Vol. 37, No. 3, pp. 843-855.

- Roll, R. (1988). 'R-squared'. The Journal of Finance, Vol. 43, No. 3, Papers and Proceedings of the Forty-Seventh Annual Meeting of the American Finance Association, Chicago, Illinois, December 28-30, 1987, pp. 541-566.

- Rosenberg, B. y MacKibben, W. (1973). 'The Prediction of Systematic and Specific and Specific Risk in Common Stocks'. The Journal of Financial and Quantitative Analysis, Vol. 8, No. 2, pp. 317-333.

- Ross, S., Westerfield, R y Jaffe, J. (2002). 'Corporate Finance'. Sixth Edition. McGraw Hill.

- Sharpe, W. (1964). 'Capital Asset Prices: A Theory of Market Equilibrium under Conditions of Risk'. The Journal of Finance, Vol. 19, No. 3, pp. 425442.

- Sunder, S. (1980). 'Stationarity of Market Risk: Random Coefficients Tests for Individual Stocks'. The Journal of Finance, Vol. 35, No. 4, pp. 883-896.

- Tobin, J. (1958). 'Liquidity Preference as Behavior Toward Risk'. Review of Economic Studies, Vol. 25, No. 2, pp. 65-86.

- Varian, H. (1992). 'Análisis Microeconómico'. Tercera Edición. Antoni Bosch.

- Vasicek, O. (1973). 'A Note on Using Cross-Sectional Information in Bayesian Estimation of Security Betas'. Journal of Finance, Vol. 8, No. 5, pp. 12331239 .

- Wells, C. (1994). 'Variable betas on the Stockholm exchange 1971-1989'. Applied Financial Economics, Vol. 4, pp. 75-92.

- West, M., Harrison, P. y Migon, H. (1985). 'Dynamic Generalized Linear Models and Bayesian Forescasting'. Journal of the American Statistical Association, Vol. 80, No. 389, pp. 94-95.

- Wong Dávila (2003). "What Factors Explain Stock Returns in Latin America? An Analysis of the Period 1995-2000". University of Frankfurt. 
- Wood, S. (2000). 'Modelling and Smoothing Parameter Estimation with Multiple Quadratic Penalties'. Journal of the Royal Statistical Society. Series B (Statistical Methodology), Vol. 62, No. 2, pp. 413-428.

- Yatchew, A. (2003). 'Semiparametric Regression for the Applied Econometrician'. Cambridge University Press. 


\section{Anexo}

\subsection{Empresas por sectores}

Empresas listadas en la BCBA entre 1994-2007 según sector al que pertenecen

\begin{tabular}{|c|c|c|}
\hline Especie & Empresa & Sector \\
\hline 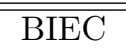 & Cerveceria Bieckert & Alimenticio \\
\hline MATE & Empresa Mate Larangeira Mendez & Alimenticio \\
\hline PATY & Quickfood & Alimenticio \\
\hline MOLI & Molinos Rio de La Plata & Alimenticio \\
\hline MORI & Morixe & Alimenticio \\
\hline CINZ & CINBA & Alimenticio \\
\hline CVZA & Compaia Industrial Cervecera & Alimenticio \\
\hline CANA & Canale & Alimenticio \\
\hline VILL & Termas Villavicencio & Alimenticio \\
\hline BAGL & Bagley & Alimenticio \\
\hline SEMI & Semino & Alimenticio \\
\hline ESME & Bodegas Esmeralda & Alimenticio \\
\hline PACL & Paclin Agropecuaria & Alimenticio \\
\hline BONA & Bonafide & Alimenticio \\
\hline SAMI & San Miguel & Alimenticio \\
\hline DACO & DACSA & Comercial \\
\hline AGRA & La Agraria & Comercial \\
\hline GOFF & Goffre, Carbone y Cia. & Comercial \\
\hline DICO & Disco & Comercial \\
\hline NOUG & Nougues & Comercial \\
\hline ACEC & ACEC & Comercial \\
\hline GRIE & Antonio Griego & Comercial \\
\hline APSA & Alto Palermo & Comercial \\
\hline REGE & Garcia Reguera & Comercial \\
\hline INAG & Insuagro & Comercial \\
\hline PATA & Patagonia & Comercial \\
\hline IGGA & Iggam & Construcción \\
\hline $\mathrm{CORC}$ & Corcemar & Construcción \\
\hline CAPU & Caputo & Construcción \\
\hline POLL & Polledo & Construcción \\
\hline NERO & Neroli & Construcción \\
\hline PATR & Patricios & Construcción \\
\hline DYCA & Dycasa & Construcción \\
\hline SALO & Ceramica San Lorenzo & Construcción \\
\hline JMIN & Juan Minetti & Construcción \\
\hline EDN & Edenor & Eléctrico \\
\hline ELEC & Electroclor & Eléctrico \\
\hline
\end{tabular}




\begin{tabular}{|c|c|c|}
\hline EMAC & Electromac & Eléctrico \\
\hline TRAN & Transener & Eléctrico \\
\hline EMDE & Emdersa & Eléctrico \\
\hline CEPU2 & Central Puerto & Eléctrico \\
\hline CTBA & Central Term. Bs As & Eléctrico \\
\hline CAPX & Capex & Eléctrico \\
\hline $\mathrm{CECO} 2$ & Central Costanera & Eléctrico \\
\hline BPAT & Banco Patagonia & Financiero \\
\hline AISA & Agritech Inversora & Financiero \\
\hline BMA & Banco Macro Bansud & Financiero \\
\hline BMAC & Banco Macro Misiones & Financiero \\
\hline BSUQ & Banco Suquia & Financiero \\
\hline BSAS & La Buenos Aires Cia. de Seguros & Financiero \\
\hline BRIO & Banco Rio & Financiero \\
\hline FRAN & Banco Frances & Financiero \\
\hline COLU & Columbia Seguros & Financiero \\
\hline GALI & Banco Galicia & Financiero \\
\hline UNIO & Union Berkley Cia. de Seguros & Financiero \\
\hline EURO & Euromayor & Financiero \\
\hline BOST & Boston cia. Argentina de Seguros & Financiero \\
\hline CITI & Telef. Hold. Arg. & Financiero \\
\hline STD & Banco Santander Central Hispano & Financiero \\
\hline BHIP & Banco Hipotecario & Financiero \\
\hline JUNC & Juncal Cia. de Seguros & Financiero \\
\hline SUPE & Banco Supervielle Societe Generale & Financiero \\
\hline VALO & Mercado de Valores & Financiero \\
\hline GGAL & Grupo Fin. Galicia & Financiero \\
\hline ELCO & El Comercio Cia. de Seguros & Financiero \\
\hline CONT & La Continental & Financiero \\
\hline GARO & Garovaglio & Holding \\
\hline PAMP & Frigorif. La Pampa & Holding \\
\hline IRSA & Irsa & Holding \\
\hline COME & Comercial del Plata & Holding \\
\hline CRES & Cresud & Holding \\
\hline FIPL & Fiplasto & Industrial \\
\hline CELU & Celulosa & Industrial \\
\hline GOTU & Gotuzzo & Industrial \\
\hline FERR & Ferrum & Industrial \\
\hline HULI & Hulytego & Industrial \\
\hline PERK & Pertrack S.A. & Industrial \\
\hline
\end{tabular}




\begin{tabular}{|c|c|c|}
\hline LONG & Longvie & Industrial \\
\hline SCHI & Schiarre & Industrial \\
\hline ESTR & Estrada & Industrial \\
\hline ROSE & Rosenbusch & Industrial \\
\hline PLAV & Plavinil Argentina & Industrial \\
\hline WELS & Wells Argentina & Industrial \\
\hline AGRO & Agrometal & Industrial \\
\hline SEVE & Sevel & Industrial \\
\hline $\mathrm{CADO}$ & Carlos Casado & Industrial \\
\hline HERE & M. Heredia & Industrial \\
\hline BGHS & Bgh & Industrial \\
\hline$\overline{\text { DELA }}$ & Della Penna & Industrial \\
\hline PIRE & Pirelli Cables & Industrial \\
\hline MASU & Massuh & Industrial \\
\hline INTA & Inta Industria Textil Argentina & Industrial \\
\hline RENO & Renault Argentina & Industrial \\
\hline PROT & Protto Hermanos & Industrial \\
\hline ZANE & Zanella Hnos. y Cia. & Industrial \\
\hline SNIA & Sniafa & Industrial \\
\hline $\mathrm{COLO}$ & Colorin & Industrial \\
\hline GRIM & Grimoldi & Industrial \\
\hline DOME & Domec & Industrial \\
\hline ALPA & Alpargatas & Industrial \\
\hline BERN & Bernardin & Industrial \\
\hline FAPL & Faplac & Industrial \\
\hline RIGO & Rigolleau & Industrial \\
\hline FRIC & Fric-Rot & Industrial \\
\hline MIRG & Mirgor & Industrial \\
\hline DECK & Decker Indelqui & Industrial \\
\hline GRAF & Grafex & Industrial \\
\hline PREN1 & Papel Prensa & Industrial \\
\hline APLA & American Plast & Industrial \\
\hline BAES & BAESA & Industrial \\
\hline TS & Tenaris & Metalúrgico \\
\hline ALUA & Aluar & Metalúrgico \\
\hline ERCA & Siderca & Metalúrgico \\
\hline MIDL & Midland & Metalúrgico \\
\hline GRAS & Ind. Siderurgicas Grassi & Metalúrgico \\
\hline ERAR & Siderar & Metalúrgico \\
\hline ACIN & Acindar & Metalúrgico \\
\hline
\end{tabular}




\begin{tabular}{|c|c|c|}
\hline TAND & Metalurgica Tandil & Metalúrgico \\
\hline BOLT & Boldt & Otros \\
\hline EDLO & Editorial Losada & Otros \\
\hline GAMI & Boldt Gaming & Otros \\
\hline EDIA & Ediar Editora & Otros \\
\hline GATI & Galimberti y Cia. & Otros \\
\hline PICA & Nobleza Piccardo & Otros \\
\hline NEPA & Negocios y Participaciones & Otros \\
\hline ATLA & Editorial Atlantida & Otros \\
\hline GCLA & Grupo Clarin & Otros \\
\hline PART & Particulares & Otros \\
\hline TABA & Tabacal & Otros \\
\hline MVIA & Metrovias & Otros \\
\hline LEDE & Ledesma & Otros \\
\hline $\mathrm{CCON}$ & Cci - Concesiones & Otros \\
\hline INTR & Introductora & Otros \\
\hline OEST & Gpo. C. del Oeste & Otros \\
\hline PAPE & La Papelera del Plata & Otros \\
\hline $\mathrm{COMO}$ & Compania Argentina de Comodoro Rivadavia & Petrolero \\
\hline YPFD & YPF & Petrolero \\
\hline METR & Metrogas & Petrolero \\
\hline CARC & Carboclor & Petrolero \\
\hline INDU & Solvay Indupa & Petrolero \\
\hline INAL & Inalruco & Petrolero \\
\hline PSUR & Petrolera del Conosur & Petrolero \\
\hline APBR & Petroleo Brasileiro & Petrolero \\
\hline ATAN & Atanor & Petrolero \\
\hline STHE & Socotherm & Petrolero \\
\hline TGNO4 & Transportadora de Gas del Norte & Petrolero \\
\hline TGSU2 & Transportadora de Gas del Sur & Petrolero \\
\hline IPAK & Ipako & Petrolero \\
\hline GBAN & Gas Natural BAN & Petrolero \\
\hline PESA & Petrobras Energia & Petrolero \\
\hline ASTR & Astra & Petrolero \\
\hline CGPA2 & Camuzzi Gas Pampeana & Petrolero \\
\hline DGCU2 & Distribuidora de Gas Cuyana & Petrolero \\
\hline PBE & Petrobras Participaciones & Petrolero \\
\hline QUES & Quimica Estrella & Petrolero \\
\hline REP & Repsol & Petrolero \\
\hline TMOV & Telefonica Moviles Arg. & Telecomunicaciones \\
\hline TEF & Telefonica & Telecomunicaciones \\
\hline TECO2 & Telecom & Telecomunicaciones \\
\hline TEAR2 & Telefonica de Argentina & Telecomunicaciones \\
\hline
\end{tabular}

Fuente: Elaboración Propia en base a Economática y BCBA. 


\subsection{Aplicaciones}

Este apartado está dedicado a esbozar algunas aplicaciones que podría tener nuestro modelo de estimación de beta usando técnicas no paramétricas.

\subsubsection{Administración de Carteras de Inversión}

En primer lugar, se ha mencionado la relevancia de beta para la confección de portafolio de acciones con fines de inversión. Cuando se evalúa la performance de un portafolio se tiene en cuenta el retorno ganado por cada uno de ellos durante un tiempo determinado, siempre y cuando dichas carteras de acciones, en este caso, tengan un riesgo similar.

Básicamente existen dos maneras de medir el riesgo de un portafolio: a través del riesgo total, es decir el desvío estándar de los retornos, ó por medio de beta. Para el interés de este trabajo, se va a considerar la segunda medida.

Generalmente los administradores de carteras suelen clasificar los portafolios en: i) riesgo bajo (beta hasta 0,7 ), ii) riesgo moderado (beta entre 0,7 y 0,9 ), y iii) riesgo alto (beta mayor a 0,9 ). La siguiente tabla clasifica los sectores en base al beta obtenido por MCO.

Cuadro A1: Clasificación de los portafolios de sectores económicos según nomenclatura de administradores de carteras.

\begin{tabular}{cc}
\hline \hline Sector & Clase de Riesgo \\
\hline Alimenticio & Moderado \\
\hline Comercial & Bajo \\
\hline Construcción & Bajo \\
\hline Eléctrico & Bajo \\
\hline Financiero & Alto \\
\hline Holding & Moderado \\
\hline Industrial & Moderado \\
\hline Metalúrgico & Alto \\
\hline Otros & Bajo \\
\hline Petrolero & Moderado \\
\hline Telecomunicaciones & Alto \\
\hline
\end{tabular}

De acuerdo al cuadro, un asesor financiero aconsejaría a un individuo con aversión al riesgo alta, invertir en una cartera de acciones de empresas de la construcción, comercio, eléctricas, ó correspondientes a otros sectores. Bajo el método de estimación aplicado en este trabajo, esta recomedación no sería adecuada.

Rellamando la ecuación $\widehat{\beta}_{s T}=\widehat{\beta}_{1 s}+\widehat{\beta}_{2 s}(T)$, la estimación por MCO considera solamente la componente paramétrica, mientras que la no paramétrica contraparte, el componente aditivo VCM, la cual fue presentada en el gráfico 1 de la sección 5. Por ejemplo, el sector de la construcción es un portafolio que 
aconsejaría un administrador de carteras a un inversor averso al riesgo. No obstante, este sector tiene un beta muy volátil. En el segundo semestre de 1997, de acuerdo al gráfico 1, la no paramétrica contraparte adicionó una buena parte del riesgo haciendo que el beta se eleve por sobre 1,1. Este portafolio se convirtió en uno extremadamente riesgoso, exponiendo al inversor a un riesgo excesivo que de ninguna forma quería asumir.

El gráfico A1 muestra la evolución aditiva de beta estimada por VCM, junto con marcadores que señalan la clase de riesgo alcanzado por la cartera.

Gráfico A1: Estimación de la parte no paramétrica de beta del sector de la construcción y desvío estándar en la estimación por VCM.

\section{Sector de la Construcción}

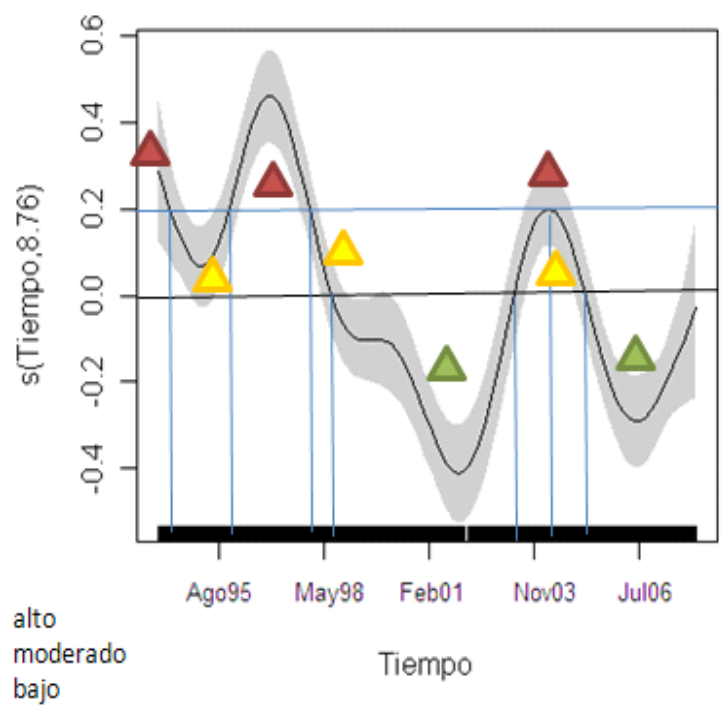

Durante el período comprendido entre el primer trimestre de 1994 y el tercer trimestre del mismo año, el portafolio del sector fue de riesgo alto según la clasificación dada por los administradores de carteras. Durante el tercer trimetre de 1994 y el cuarto trimestre de 1995 el sector fue de riesgo moderado. El sector soportó un riesgo alto para los dos años posteriores. Entre el cuarto trimestre de 1997 y el tercer trimestre de 1998, el portafolio fue de riesgo moderado. Luego, acontenció un largo período que coincide con lo que el administrador hubiese recomendado al inversor temeroso; el portafolio fue de riesgo bajo hasta el segundo trimestre de 2003. Desde entonces el sector fue de riesgo moderado alcanzando incluso, nuevamente, alto riesgo en el segundo trimestre de 2004 . Desde comienzo de 2005 y hasta el final del período de la muestra el portafolio del sector de la construcción fue de riesgo bajo. 
Por lo tanto, es dable no considerar el valor de beta, estimado por MCO, como ciencia cierta para recomendar portafolios en base al riesgo.

\subsubsection{Valuación de empresas}

Otra de las aplicaciones más comunes de beta reside en la valuación de empresas. El costo promedio ponderado del capital (WACC) es una medida utilizada para estimar el costo de capital de una empresa. Esta medida también es utilizada para la evaluación de proyectos. En este caso, el costo de oportunidad del capital utilizado debe reflejar el costo promedio ponderado de los distintos tipos de fondeo utilizado por la empresa, siempre que no cambie su estructura de capital.

Dentro de esta técnica, el beta juega un rol central en la estimación del costo de oportunidad de las utilidades retenidas por la empresa. Esta es la tasa de retorno exigida por los inversores en acciones de la propia empresa o en empresas con similares unidades de negocios.

Como ejemplo tomamos el caso de la empresa Edenor, del sector eléctrico, la cual realizó su oferta pública de acciones en Mayo de 2007. Un mes después, el banco de inversión Citigroup emitió un informe de valuación y recomendación a sus clientes sobre las acciones de la empresa. Expuso un modelo de flujo de fondos descontados y para ello utilizó un WACC de 12,2\% tomado a partir de: i) un ratio de deuda a capital de $32,6 \%$, ii) una tasa libre de riesgo de $4,7 \%$, iii) un premio por riesgo sistémico argentino de 300 puntos básicos, iv) un premio por riesgo para las acciones argentinas de $6,8 \%$, y v) un beta de 1,09 correspondiente a la empresa Pampa Holding, con una importante presencia en el sector eléctrico argentino.

La estimación de beta se basó en la metodología de MCO para un período de 2 años bajo el MIS, tomando como mercado al índice Merval ${ }^{2122}$. De esta manera, el cálculo del costo de financiamiento por acciones, y consecuentemente el WACC se observan en el cuadro A2.

\footnotetext{
${ }^{21}$ Pampa Holding compró el capital de Frigorífico La Pampa a fines de 2005 para transformar su unidad de negocio al sector eléctrico. Tomar una ventana de dos años, y no más, estaba contando por este hecho.

${ }^{22}$ Bases de datos como Bloomberg, Reuters, Economática y IAMC presentan valores de beta obtenidos a partir de MCO en una ventana generalmente de 60 meses. Estos datos son ampliamente usando por los analistas financieros.
} 
Cuadro A2: Costo de financiamiento por acciones y costo promedio ponderado del capital según metodología y datos de Citigroup.

\begin{tabular}{cc}
\hline \hline Beta & $109 \%$ \\
\hline Tasa Libre de Riesgo & $4,70 \%$ \\
\hline Premio por Acciones ARG & $6,80 \%$ \\
\hline Premio Riesgo Soberano & $3 \%$ \\
\hline Costo de Fin. Acciones & $15,11 \%$ \\
\hline Premio por Riesgo Deuda & $4,70 \%$ \\
\hline Costo Deuda desp. Imp. & $6,10 \%$ \\
\hline Deuda a Capital & $32,60 \%$ \\
\hline WACC & $12,17 \%$ \\
\hline \hline
\end{tabular}

Tomando los mismos valores que utilizó Citigroup pero utilizando el valor de beta del sector eléctrico computado por la estimación no paramétrica, es posible estimar el WACC de manera más consistente.

Cuadro A3: Costo de financiamiento por acciones y costo promedio ponderado del capital según metodología y datos de Citigroup, excepto beta calculado por VCM.

\begin{tabular}{cc}
\hline \hline Beta & $48,94 \%$ \\
\hline Tasa Libre de Riesgo & $4,70 \%$ \\
\hline Premio por Acciones ARG & $6,80 \%$ \\
\hline Premio Riesgo Soberano & $3 \%$ \\
\hline Costo de Fin. Acciones & $11,03 \%$ \\
\hline Premio por Riesgo Deuda & $4,70 \%$ \\
\hline Costo Deuda desp. Imp. & $6,10 \%$ \\
\hline Deuda a Capital & $32,60 \%$ \\
\hline WACC & $9,42 \%$ \\
\hline \hline
\end{tabular}

El costo de financiamiento por acciones se reduce más de 400 puntos básicos y, consecuentemente, el WACC se reduce en 275 puntos básicos. Estos nuevos valores son utilizados en el modelo de flujos de fondos descontados. Tomando las proyecciones de flujos de fondos de Citigroup pero recalculando a partir de los nuevos valores obtenidos, el valor presente neto termina aumentando $42 \%$, mientras que el precio objetivo de la acción sube $43 \%$. El beta no paramétricamente calculado corrige severamente las estimaciones realizadas por Citigroup. 